\title{
A General Method to Analyze the Thermal Performance of Multi-Cavity Concentrating Solar
}

\section{Power Receivers}

Thermal Analysis, Multi-Cavity Receiver, Austin Fleming ${ }^{\mathrm{a}}$, Charles Folsom ${ }^{\mathrm{a}}$, Heng Ban ${ }^{\mathrm{a},{ }^{*}}$, and Zhiwen $\mathrm{Ma}^{\mathrm{b}}$

${ }^{a}$ Mechanical \& Aerospace Engineering Department, Utah State University, Logan, UT 84322-4130

${ }^{\mathrm{b}}$ National Renewable Energy Lab, Golden, CO 80401

*+1.435.797.2098 heng.ban@ usu.edu, 4130 Old Main Hill, Logan, UT 84322-4130

\begin{abstract}
Concentrating solar power (CSP) with thermal energy storage has potential to provide grid-scale, ondemand, dispatachable renewable energy. As higher solar receiver output temperatures are necessary for higher thermal cycle efficiency, current CSP research is focused on high outlet temperature and high efficiency receivers. The objective of this study is to provide a simplified model to analyze the thermal efficiency of multi-cavity concentrating solar power receivers. The model calculates an optimal aperture flux that maximizes the local efficiency, constrained by a maximum receiver working temperature. Using this flux, the thermal efficiency, receiver temperature, and heat transfer fluid (HTF) temperature are calculated based upon an optimized flux distribution. The model also provides receiver design and HTF heat transfer requirements to achieve the necessary overall thermal efficiency. From the results, possible HTFs can be investigated to determine which ones are feasible. A case study was performed on a multicavity tube receiver design to demonstrate the use of the model. The case study receiver design had an effective absorptivity of $99.8 \%$, and was modeled with conservative values for thermal constraints. It was found that a HTF with a minimum convection coefficient between $250-500 \mathrm{~W} \cdot \mathrm{m}^{-2} \cdot \mathrm{K}^{-1}$, depending on the convective heat transfer to the environment, is necessary to achieve a thermal efficiency greater than $90 \%$ for the receiver. The general model can provide a design guideline for attainable thermal efficiencies of multi-cavity concentrating solar power receivers given thermal constraints and heat transfer conditions.
\end{abstract}

Key words: Concentrated Solar Power, Multi-cavity Receiver, Thermal Analysis 


\section{Introduction}

The key challenge to implementing grid-scale concentrating solar power (CSP) is to reduce its cost and couple it with thermal energy storage to offer on-demand, dispatachable electricity. Research has been conducted on the logistics and cost benefits of coupling CSP with thermal energy storage [1-3]. Through the SunShot Initiative, the US-DOE has identified areas of research to reduce the cost of CSP to compete with coal and nuclear power at a cost of $6 \notin / \mathrm{kWh}$ [4]. These areas of research include: improved solar fields with respect to efficiency and cost, more advanced heat transfer fluids capable of a wide operating temperature range, improved thermal energy storage (TES) capability, cooling technology, and advanced receiver designs capable of high thermal efficiency and higher heat transfer fluid (HTF) outlet temperatures than currently available. The levelized cost of energy from CSP can be decreased by reducing the manufacturing costs and increasing the efficiency of CSP plants.

The current state-of-the-art receiver is considered to be a panel receiver consisting of tubular panels with molten salt as the HTF. These have proven to be reliable and are most common in operating CSP plants today, but to date they are limited to less than $90 \%$ thermal efficiency and outlet temperatures of $565^{\circ} \mathrm{C}[5,6]$. While these CSP plants have improved, they still are not cost competitive to provide mainstream power production.

Many CSP power tower designs have been evaluated both with simulations and experiments. These include tubular, cavity, multi-cavity, volumetric receivers and direct absorbing receivers with working fluids including but not limited to: steam, molten salt, molten metal, gas, and particles [7-13]. Most commonly, the receiver analysis is conducted using detailed analysis tools such as computational fluid dynamics (CFD), finite element modeling, and Monte Carlo ray tracing. While these techniques are accurate, they are computationally expensive and often impose restrictions on the size of the geometry that can be modeled. This often limits the analysis to localized regions in the receiver. Some receiver designs can be approximated as an isothermal receiver; in reality however, there is a temperature distribution that varies throughout the receiver due to the varying local flux intensities and HTF temperatures. Furthermore, if the overall receiver efficiency is of interest, it is often difficult to obtain using the detailed computational analysis techniques discussed above because they require modeling the entire receiver which is computationally expensive.

This study focuses on developing an analysis technique that can quantify the thermal performance of CSP receivers quickly without the need for extensive detailed computational analysis. The technique developed will also allow for quantitative comparisons between receiver designs using different geometries, working fluids, and sizes. An application of this technique will be demonstrated on a case study for a receiver design to show how it can be applied to a specific geometry and operating conditions. 
In this case study it will be demonstrated how more accurate methods, such as Monte Carlo ray tracing

2 and CFD, can be incorporated in the technique to increase the accuracy.

\section{2. Method and Procedure}

The technique development is generalized as much as possible to be applicable to many multi-cavity receiver geometries, HTFs, and operating conditions. The motivation for this technique is to develop a method with the ability to rapidly analyze the thermal performance of a CSP receiver design. Providing this ability enables engineers and researchers to easily determine the feasibility of a receiver design to meet design requirements. Furthermore, it can be used to guide researchers to focus on specific areas where design improvements can be made, or which components the receiver performance is most sensitive to. The technique development process will first establish the assumptions and constraints of the procedure. Next, the analysis procedure will be discussed along with the motivation for each result. Finally, the implications and applications of the technique will be discussed.

The only receiver cavity geometry constraint for the technique presented here is an indirect absorbing receiver in which the aperture area $\left(A_{\text {aper }}\right)$ of the receiver and the heat transfer area of the HTF $\left(A_{h t f}\right)$ can be clearly defined, such that $A_{h t f} \geq A_{\text {aper }}$. If the receiver consisted of a flat plate with solar flux incident on one side and the HTF on the other, then the aperture area and heat transfer area would be the same. However, in other instances where the surface absorbing radiation is not at the aperture of the receiver, a larger $A_{h t f}$ can be obtained. For example, if the absorbing surface was a series of parallel tubes (diameter $D$ and length $L$ ) with the edges touching, the aperture area for one tube would be $D \cdot L$, whereas the heat transfer area would be $\pi D L / 2$, since only one side of the tube is illuminated. High thermal efficiency is possible with a multi-cavity receiver by utilizing a heat transfer area much larger than the aperture area. This results in a lower surface temperature by spreading the flux across the heat transfer area thereby reducing the radiation and convection losses to the environment. In this study nothing else is specified about the geometry of receivers other than the ratio of $A_{h t} f A_{\text {aper }}$ (referred to as the area ratio, $A_{\text {ratio }}$, for the remainder of the paper). By keeping the analysis general, the results are comparable to many different designs of surface absorbing receivers.

This simplified model evaluates the energy balance in a small control volume (CV) at the aperture of the receiver, with no consideration to the geometry of the receiver (see Figure 1). Since the CV being evaluated is small, the aperture flux, receiver temperature, and HTF temperature can be considered a constant throughout the $\mathrm{CV}$. In the $\mathrm{CV}$, the working fluid is assumed to be the same temperature as it was at the inlet. A grid convergence study was performed on the size of the CV to ensure the accuracy of the constant $T_{h t f}$ and $T_{R}$ inside the CV assumption. Eqn. (1) is therefore derived by performing an energy 
1 balance throughout the $\mathrm{CV}$, assuming energy in as flux from the solar field and losses due to re-radiation

2 of the receiver, natural convection losses to the environment, and energy transferred to the HTF.

$$
A_{\text {aper }} q_{\text {aper }}^{\prime \prime} \eta_{\text {absorb }}=\varepsilon_{\text {eff }} \sigma A_{\text {aper }}\left(T_{R}^{4}-T_{\text {sur }}^{4}\right)+h_{\text {amb }} A_{\text {aper }}\left(T_{R}-T_{a m b}\right)+h_{h t f} A_{h t f}\left(T_{R}-T_{h t f}\right)
$$

Here, $A_{\text {aper }}$ is the aperture area of the receiver, $q_{a p e r}^{\prime \prime}$ is the flux from the solar field, $\eta_{a b s o r b}$ is the

absorption efficiency of the receiver, $\varepsilon_{\text {eff }}$ is the effective emissivity of the receiver defined based on the aperture area, $\sigma$ is Boltzmann's constant, $T_{R}$ is the temperature of the receiver in the CV, $T_{\text {sur }}$ is the 6 temperature of the surroundings, $h_{a m b}$ is the convection coefficient to the environment, $T_{a m b}$ is the ambient 7 air temperature of the environment, $h_{h f f}$ is the convection coefficient between the receiver and the HTF, $8 A_{h t f}$ is the heat transfer surface area to the HTF, and $T_{h t f}$ is the temperature of the HTF in the CV.

9 The effective emissivity can be calculated by determining the amount of radiation emitted from the 10 cavity, and then calculating what the emissivity would be if all of this radiation was emitted from a flat 11 plate covering the aperture of the cavity. There are many techniques that can be used to calculate this 12 radiation exchange, and the appropriate one depends on the cavity shape, size, and material. In this study 13 a conservative approach has been taken to estimate the effective emissivity as 1 . This can be a good 14 approximation for many cavities since a cavity with a small hole is often used to approximate black body 15 emission.

16 In certain scenarios it is possible for the receiver to exchange radiation with the surrounding at one 17 temperature and the receiver to exchange heat through convection with the surrounding fluid at a different 18 temperature. For this reason, in the analysis a $T_{a m b}$ and $T_{\text {sur }}$ have been used to have the ability to set these 19 convection and radiation exchange temperatures, respectively.

With the heat transfer parameters given, the only unknown that needs to be solved for is the temperature of the receiver $\left(T_{R}\right)$. With these conditions, the local thermal efficiency $\left(\eta_{R}\right)$ of the receiver, i.e. the efficiency at a specific $T_{h t f}$ and $q_{a p e r}^{\prime \prime}$, is calculated as:

$$
\eta_{R}=\frac{h_{h t f} A_{h t f}\left(T_{R}-T_{h t f}\right)}{A_{\text {aper }} q_{\text {aper }}^{\prime \prime}} .
$$

The temperature rise of the heat transfer fluid in the control volume can be calculated by

$$
\eta_{R} q_{\text {aper }}^{\prime \prime} A_{\text {aper }}=\dot{m} C_{p}\left(T_{h t f, \text { out }}-T_{h t f, \text { in }}\right) \text {, }
$$

24 where $\dot{m}$ is the mass flow rate of the HTF in the $\mathrm{CV}, C_{p}$ is the specific heat of the HTF, and the in and 25 out subscripts refer to the inlet and outlet temperatures of the HTF, respectively. This equation can be 26 rewritten as

$$
T_{h t f, \text { out }}=T_{h t f, \text { in }}+\frac{\eta_{R} q^{\prime \prime}{ }_{\text {aper }}}{K},
$$

27 where $K$ is a constant that equals 


$$
K=\frac{\dot{m} C_{p}}{A_{\text {aper }}}
$$

The use of $K$ in the model allows the thermal analysis to be independent of receiver geometry and HTF properties. It should be noted that by using the constant $K$, the overall size of the entire receiver is not considered, although the size of the entire receiver will affect the overall cost, which is not considered in this study.

In this study it is assumed that the outlet temperature can always be reached with a given receiver design, provided that there is sufficient flux. This can be done in reality by, increasing the length of the HTF flow path (larger receiver or multiple pass), reducing the mass flow rate until the output temperature is reached, or utilizing a HTF with a lower specific heat such that it heats up more with the same amount of energy. An artifact of this assumptions shows up in the value for $K$ used in the analysis. Since the value of $K$ contains the $\mathrm{A}_{\text {aper }}$ of the $\mathrm{CV}$, the value of $K$ determines the number of control volumes in the analysis. From a grid convergence study it was determined for a receiver with inlet and outlet of $500 \mathrm{~K}$ and $1100 \mathrm{~K}$ respectively that $\sim 10,000$ control volumes are necessary to obtain an efficiency with a difference of $0.01 \%$ compared to running with orders of magnitude more control volumes. In each simulation, based on an initial evaluation for of the receiver efficiency and aperture flux, a value of $K$ is calculated such that $\sim 10,000$ control volumes will be used in the analysis. This corresponds to a temperature rise of approximately $0.06 \mathrm{~K}$ over each $\mathrm{CV}$ given the inlet and outlet temperatures defined above.

The thermal efficiency of the receiver as a whole can be determined by integrating the local efficiency multiplied by the local flux over the whole receiver. This integration is conducted by summing the energy transferred to the HTF over all CV's divided by the sum of the flux incoming to the receiver over all CV's which can be shown as

$$
\eta_{R E C}=\frac{\sum \eta_{i} q_{i}^{\prime \prime}}{\sum q_{i}^{\prime \prime}}
$$

21 where $\eta_{\mathrm{i}}$ refers to the local efficiency of the $\mathrm{i}^{\text {th }}$ control volume and $q_{i}^{\prime \prime}$ represents the aperture flux of the $22 i^{\text {th }}$ control volume.

\section{Results and Discussion}

\section{3.1. Generic Model}

25 It should be noted that the $A_{\text {ratio }}$ only shows up multiplied by $h_{h t f}$ in Eqn. (2). Therefore doubling the 26 area ratio would have the same effect as doubling the $h_{h t f}$. The ratio $h_{h t f} A_{h t f} / A_{a p e r}$ will be referred to as 27 the $h A_{\text {ratio }}$ throughout the remainder of this paper. Also the efficiency calculated for the CV will be 28 referred to as a local efficiency in this paper, since it corresponds to a specific $T_{h t \text {. }}$ Extending this idea, the 
local efficiency can be determined throughout the entire receiver by varying $T_{h t f}$ from the input temperature to the output temperature of the receiver. This analysis has been performed on a receiver with an inlet temperature of $400 \mathrm{~K}$ and outlet temperature of $1000 \mathrm{~K}$ with an $h A_{\text {ratio }}$ varying from 1000-5000 $\mathrm{W} \cdot \mathrm{m}^{-2} \cdot \mathrm{K}^{-1}$. A convection coefficient between the receiver and ambient environment of $25 \mathrm{~W} \cdot \mathrm{m}^{-2} \cdot \mathrm{K}^{-1}$ has been chosen. This value is a conservative estimate based on other studies which have used similar values as a baseline for convection losses $[8,14,15]$. More accurate values can be found through experimentation or computational analysis for more specific receiver designs. For this model a conservative value of unity for $\varepsilon_{\text {eff }}$ was chosen. This value can be calculated based on material properties and view factors when a specific receiver cavity geometry and properties are analyzed. However, for geometries with large $A_{\text {ratio }}$ 's this value will approach unity due to the black body emission principle. As indicated in Figure 2, the local efficiency of the receiver increases with increasing $h A_{\text {ratio }}$ values, but decreases with increasing temperature of the HTF. From this, the total receiver thermal efficiency can be obtained by integrating the local receiver efficiency and aperture flux over the whole receiver. For these three cases the overall receiver efficiencies, starting with an inlet HTF temperature of $400 \mathrm{~K}$ and ending when the HTF temperature reached $1000 \mathrm{~K}$ were: $0.717,0.898$, and 0.923 for a $h A_{\text {ratio }}$ of 1000,3000 , and $5000 \mathrm{~W} \cdot \mathrm{m}^{-2} \cdot \mathrm{K}^{-1}$, respectively.

While the results in Figure 2 provide an understanding of how $h A_{\text {ratio }}$ values affect the local efficiency, the understanding is limited to single aperture flux of $1 \mathrm{MW} / \mathrm{m}^{2}$. It is of interest to engineers to understand how efficiency is affected by the aperture flux and how to optimize efficiency by altering the aperture flux. To answer this question, the local efficiency of a receiver was calculated for a range of $h A_{\text {ratio }}$ values, aperture flux values, and working fluid temperatures. The results show that given a $h A_{\text {ratio }}$ value, there is an optimal value for aperture flux to maximize thermal efficiency, which is illustrated in Figure 3. These results also show that while an optimum flux exists to maximize efficiency for a specific $h A_{\text {ratio }}$, the opposite is not true. There is not an optimum $h A_{\text {ratio }}$ given an aperture flux, except the general rule of the higher, the better. It should also be noted that as the HTF temperature increases, the optimal value flux becomes higher. Therefore, for a given receiver design, the optimized flux distribution would be low at the HTF inlet of the receiver, and increase monotonically toward the exit.

While it is of use to know how the efficiency varies locally, it is typically more important to know the total thermal efficiency of the receiver. To provide this information, a receiver was assumed to have an inlet temperature of $400 \mathrm{~K}$ and outlet temperature of $1000 \mathrm{~K}$ and the receiver efficiency was calculated for a range of aperture flux and $h A_{\text {ratio }}$ values. For this study, the aperture flux was assumed to be uniform over the receiver. This was conducted for three values of convection coefficients to the environment of 0 , 25 , and $50 \mathrm{~W} \cdot \mathrm{m}^{-2} \cdot \mathrm{K}^{-1}$. These results can provide CSP design engineers with guidelines of what overall 
receiver thermal efficiencies are possible given a uniform aperture flux and inlet and outlet temperatures over a range of $h A_{\text {ratio }}$ values, as can be seen in Figure 4.

As discussed with Figure 3, there is an optimal flux at any given location in the receiver that maximizes the efficiency at a specific $h A_{\text {ratio. }}$. Therefore, it is important to know how the flux should be distributed across the face of the receiver to maximize the overall receiver efficiency, which may be possible with improvements in heliostat aiming methods [16-19]. In order to determine this optimal flux distribution, the local receiver efficiency was calculated at each location for a range of aperture flux values. Then at each location, the flux that provided the maximum efficiency is chosen. Performing this same analysis over all locations in the receiver provides the optimal flux distribution throughout the receiver. This optimal flux distribution and corresponding receiver temperature and local efficiency can be seen in Figure 5. It is reiterated that as the temperature of the HTF increases, the required flux must also increase, but the efficiency will decrease. The decrease in efficiency is inevitable due to the increase in temperature, but the gradient is minimized by optimizing the flux. The overall thermal efficiency for this case is $89.72 \%$.

Often, this scenario is not possible due to limitations on the maximum operating temperature of the receiver material. The maximum operating temperature of the receiver design can be limited by many factors related to both mechanical properties (thermal stress, fatigue, creep) and chemical properties (oxidation/corrosion). This limitation is dependent on the receiver material, geometry and HTF. While, in general, each receiver design requires extensive analysis to determine which factor limits the operating temperature, it is outside of the scope of this research. It is assumed here that an estimation for the maximum working temperature can be found based on design. In practice, a more detailed analysis, such as finite element analysis, can be conducted to obtain a better approximation for the maximum operating temperature.

This leads to the next point of interest for the design engineer, "What is the optimal flux distribution for the receiver that maximizes thermal efficiency without exceeding the maximum working temperature of the receiver?" To answer this question, the optimum receiver flux was determined at each location in the receiver with the constraint that the maximum receiver temperature cannot be exceeded. The results from this study show the same initial trend as Figure 5, until the maximum receiver temperature is reached. At that point, the optimum flux begins to decrease while maintaining the receiver at its maximum working temperature. The efficiency at that location also begins to drop off more rapidly than previously without the material constraint. These results can be seen in Figure 6. The overall thermal efficiency in this case is $89.57 \%$ which is only a $0.15 \%$ drop in efficiency by ensuring that the temperature of the receiver does not exceed its working limit. 
While knowing what the optimal flux distribution looks like is important, it is of more interest to a 2 design engineer to understand how the material limitations affect the total receiver efficiency. To evaluate 3 these effects, a receiver HTF inlet and outlet temperatures were assumed to be $400 \mathrm{~K}$ and $1000 \mathrm{~K}$, 4 respectively. The flux was optimized throughout the receiver with the constraint of the maximum working 5 temperature of the receiver. This was conducted for a range of $h A_{\text {ratio }}$ values and a range of convection 6 coefficients with the environment. From these results, the maximum receiver efficiency can be 7 determined given a value of $h A_{\text {ratio }}$, maximum receiver working temperature, and natural convection 8 coefficient, as seen in Figure 7.

9 The results presented above provide the thermal efficiency for CSP receivers through a range of 10 designs parameters and operating conditions. Furthermore, a simple method to determine thermal 11 efficiency of a CSP receiver with material property limitations has been provided. Throughout this 12 analysis, the only design considerations assumed about the receiver is that it is a surface absorbing 13 receiver and that there exists some $h A_{\text {ratio }}$. From these results, it is clear that an increase in the HTF 14 convection coefficient or the $A_{\text {ratio }}$ significantly improves the thermal efficiency of the receiver. This is the 15 driving motivation for this research in multi-cavity receivers.

While there are many techniques to analyze CSP receivers, there is a strong need to couple simplified models (such as the one presented above) and high fidelity models (such as Monte Carlo Ray 18 Tracing, Discrete Ordinates, Computational Fluid Dynamics, etc.). In the following section, we will perform a case study on a given design for a CSP receiver. The analysis technique couples together both the simplified and high fidelity techniques. This allows for accurate modeling to be performed in a timeframe that allows for optimization of receiver performance and design.

\subsection{Case Study}

This case study will demonstrate how the model described above can be used to analyze a specific multi-cavity CSP receiver design. This case study will begin by defining a geometry of interest in which the Monte Carlo ray tracing program SolTrace [20] will be used to determine the overall absorption 
The receiver geometry being evaluated in this case study is a multi-cavity receiver in which the cavities are in the shape of hexagon tubes. The main body of the tubes are approximately 2 inches $(50.8$ $\mathrm{mm})$ high by 1 inch $(25.4 \mathrm{~mm})$ wide. The tapered entrance region is approximately 2.3 inches $(58.4 \mathrm{~mm})$ 4 high by 1.3 inches $(33 \mathrm{~mm})$ wide at the aperture. The entrance region is approximately 1.6 inches (40 $\mathrm{mm})$ long with the total length of the tube of 10 inches $(254 \mathrm{~mm})$. The tubes have a $15^{\circ}$ angle to better align the tube axis with the incident light from the heliostats. In this design, it is assumed that the HTF flows on the outside of the tubes (similar to flow over a bank of tubes) from the top of the receiver down. This design results in an $A_{\text {ratio }}$ of 21.65. The geometry can be seen in Figure 8.

To determine the loss due to reflected solar flux, SolTrace was used. In this study, a heliostat field layout from the National Solar Thermal Test Facility (NSTTF) site was used with heliostats and specific aim points selected to achieve a uniform flux distribution. In a study for power generation, the heliostat field layout will play a role in receiver design as well as the range of flux intensities that the field is capable of. Additionally, the heliostat field will determine the ability to control the flux intensity spatially on the receiver aperture. However, these heliostat design considerations are outside the scope of work for this study.

The motivation for this specific multi-cavity receiver design is to have light that is reflected on first contact with the cavity propagate into the receiver where it will either be absorbed or reflected again further into the cavity. This design allows for effective absorptivity values to be much larger than the absorptivity of the receiver material itself (blackbody effect) [13]. Additionally, this reduces the maximum flux on the receiver by spreading it out throughout the cavity instead of absorbing all of the radiation at the aperture. This result can be seen both qualitatively and quantitatively in Figure 9. For this simulation, the aperture flux was $1.27 \mathrm{MW} / \mathrm{m}^{2}$, the maximum flux on the receiver cavity was approximately 0.22 $\mathrm{MW} / \mathrm{m}^{2}$, the minimum was $10 \mathrm{~kW} / \mathrm{m}^{2}$, and the majority of the receiver cavity is between $100-200 \mathrm{~kW} / \mathrm{m}^{2}$.

From Figure 9, it is easy to see that the flux has been effectively distributed through the cavity of the receiver. However, it should also be noted that the flux distribution is not uniform throughout the cavity. While this is not necessarily a problem for operation, it needs to be a consideration in the modeling conducted with the simplified model. If it was possible to evenly spread the aperture flux ( $\left.q_{a p e r}^{\prime \prime}\right)$ throughout the cavity, then the ratio of the aperture flux to the maximum flux $\left(q_{\max }^{\prime \prime}\right)$ must be equal to the $A_{\text {ratio. }}$ The closer the ratio of the flux is to the $A_{\text {ratio }}$, the more uniform the distribution is on the heat transfer surface of the receiver. If there is a cavity that is 10 meters deep, but no solar flux reaches the last 6 meters (resulting in a flux ratio much greater than the area ratio), then the last 6 meters should not be included as a heat transfer surface area and therefore not included in the $A_{\text {ratio }}$ calculation. In the analysis process, it can be difficult to determine at what point the cavity surface is no longer an effective heat transfer surface area. There are many legitimate methods to determine the appropriate area to use in 
calculations, however, in an effort to ensure repeatable and conservative results, the authors used the ratio

2 of $q_{\text {aper }}^{\prime \prime} / q_{\max }^{\prime \prime}$. This ensures conservative results, rather than attempting to determine the area of effective

3 heat transfer on a case by case basis. Additionally, it is recommended that the flux distribution of the

4 receiver, similar to Figure 9, be used to ensure a good distribution of flux throughout the cavity.

Often the two most important flux distribution parameters are the ratio of the aperture flux to the maximum flux on the receiver and the absorption efficiency. These two parameters determine how much light is absorbed and how well it is spread throughout the cavity. The quality of flux spreading throughout 8 the cavity will provide an estimate for the effective $A_{\text {ratio }}$ of the multi-cavity receiver. For this case study, a 9 range of optical parameters were chosen since no specific receiver material has been determined at this 10 point. For this reason, the SolTrace program was used to simulate a range of receiver material reflectivity 11 and specularity error values. The results in Figure 10 show that $>97 \%$ absorption efficiency can be 12 obtained over a large range of reflectivities and specularity error values. Furthermore, from Figure 10 it 13 can be seen the absorption efficiency monotonically decreases with increase in reflectivity. It can be seen 14 that less specularity error leads to higher absorption efficiency. This is due to the fact that light that is 15 reflected more diffusely has a higher probability of being reflected back to the environment and not 16 contacting the cavity again. This is accurate for cavities whose axis is predominately aligned with the 17 direction of the incoming flux.

18 Figure 11 shows the ratio of aperture flux to maximum flux in the receiver. This is an important plot 19 because it defines the Aratio values for different cavity reflectivities and specularity errors. From this plot 20 it can be seen that generally the flux ratio increases with reflectivity and specularity. However, there are 21 scenarios where this is not the case, and these exceptions typically depend on the location of the 22 maximum flux. For example, if the location of maximum flux was at the back of the cavity, and by 23 decreasing the specularity error, it is possible that more solar flux could reach the back of the cavity, 24 therefore increasing the maximum value. Similar abnormalities can be seen when a change in either 25 reflectivity or specularity error leads to a change in the location of the maximum flux.

Now, we can use these ray tracing results to define an effective $A_{\text {ratio }}$ and reflection loss to be used in 26 the rest of the analysis. Additionally, a range of convection coefficients to the environment need to be 28 assumed, along with the surrounding temperatures for radiation. To ensure a conservative estimation for thermal efficiency, surrounding radiation surfaces and fluid temperature are assumed to be $300 \mathrm{~K}$. The convection coefficient of the heat transfer from the receiver to the ambient air is one of the most difficult values to quantify in the analysis. While detailed CFD analysis can yield accurate values for a given scenario, the results can only be as accurate as the inputs allow. While much work has been conducted on quantifying natural convection loss in cavity receivers [15, 25-28], these results are highly dependent on 34 the direction and magnitude of any incident wind on the receiver. For this reason, a range of possible 
ambient convection coefficients will be analyzed. This will provide information on how the receiver

2 performance could be altered in varying weather conditions. Finally, the emissivity of the receiver is

3 assumed unity due to the blackbody emitter principle of a cavity. At this point in the case study, we will

4 assume that the receiver material is stainless steel, which will give a reflectivity of approximately 0.5 and

5 we will choose the case where our specularity error is $600 \mathrm{mRad}$. From Figure 10 and Figure 11, this 6 gives us an absorption efficiency of $99.8 \%$ and an effective $A_{\text {ratio }}$ of 7.01. For material limitations, we will 7 assume a maximum receiver material working temperature of $1200 \mathrm{~K}$. The flux distribution on the 8 receiver will be optimized to allow for the highest efficiency possible, as discussed above. Since the HTF 9 has not been set, a range of HTF convection coefficients will be analyzed. This will allow for the 10 comparisons of different HTFs in the analysis of this multi-cavity receiver.

11 At this point in the case study, all heat transfer parameters have been set with the exception of the 12 convection coefficients for receiver-environment and receiver-HTF. In practice, it is difficult to accurately 13 determine the receiver-environment convection coefficient. This is predominately due to the varying wind conditions combined with natural convection currents. However, a range can be determined based on the 15 range of typical convection coefficients for air. In this study a range between $0-50 \mathrm{~W} \cdot \mathrm{m}^{-2} \cdot \mathrm{K}^{-1}$ was chosen. 16 Likewise, a range of receiver-HTF convections coefficients were chosen which can be representative of 17 different HTF's and/or different flow conditions of the HTF. Using these ranges of values for receiver18 environment and receiver-HTF convection coefficients, an analysis on the thermal efficiency can be conducted. From the analysis it can be determined that efficiencies $>90 \%$ need a minimum $h_{h t f}$ of 250 $\mathrm{W} \cdot \mathrm{m}^{-2} \cdot \mathrm{K}^{-1}$, and may require an $h_{h t f}$ of up to $500 \mathrm{~W} \cdot \mathrm{m}^{-2} \cdot \mathrm{K}^{-1}$, if the ambient convection coefficient is 50 $\mathrm{W} \cdot \mathrm{m}^{-2} \cdot \mathrm{K}^{-1}$.

If the designer imposes a minimum thermal efficiency for the receiver, then Figure 12 can be used to determine what convection coefficient is necessary for the HTF. Additionally, this HTF must be capable of meeting the output temperature of the receiver. A compilation of common working fluids and their respective convections coefficients and working temperature ranges have been compiled in Table 1. While this list is not exhaustive of all possibilities, it provides a qualitative reasoning process for design. With the design requirements imposed on this case study and only considering HTFs within the scope of Table 29 1, we can determine that only sand, air, and helium would be capable of meeting the $>900^{\circ} \mathrm{C}$ outlet 30 temperature requirements of the receiver design. If we impose a $>90 \%$ thermal efficiency requirement on 31 the receiver design, then Figure 12 can be utilized to determine what HTF convection coefficient is 32 necessary to meet this requirement under the range of natural convection losses. For the design in this 33 case study, it has been determined that a minimum convection coefficient of $250 \mathrm{~W} \cdot \mathrm{m}^{-2} \cdot \mathrm{K}^{-1}$ is required to 
meet these requirements. Furthermore, using Table 1, sand would be the only HTF capable of meeting the heat transfer requirements to meet a $90 \%$ thermal efficiency for this design.

Table 1. Table of known heat transfer fluids (salts, metals, particles, oils, gases) properties [29-33]

\begin{tabular}{cccc}
\hline HTF & $\begin{array}{c}\text { Convection Coefficient } \\
\left(\mathbf{W} \cdot \mathbf{m}^{-2} \cdot \mathbf{K}^{-\mathbf{1}}\right)\end{array}$ & $\begin{array}{c}\text { Working Temp. } \\
\text { Range }(\mathbf{K})\end{array}$ & Notes \\
\hline Sodium & $22,700-45,400$ & $423-863$ & \\
Nitrate Salts & $5,700-11,400$ & $553-883$ & $10 \mathrm{MPa}$ \\
Water (Saturated) & $7,400-68,100$ & $273-588$ & $10 \mathrm{MPa}$ \\
Steam & $1,700-8,500$ & $588-813$ & Caloria HT-43 \\
Oil & $570-5,700$ & $<588$ & Olivine sand \\
Sand & $50-900$ & $>1300$ & \\
Air & $25-250$ & $>1300$ & \\
Helium & $25-250$ & $>1300$ & \\
\hline
\end{tabular}

4

As in any design process, an iterative analysis should be conducted to optimize the design. For the sake of brevity, the full optimization process will not be discussed, but for this receiver it is clear that if the effective $A_{\text {ratio }}$ could be increased, the efficiency could be significantly increased. From Figure 9, it should be noted that the maximum flux was at the transition between the entrance region of the tube and the body. If a design modification was made that reduced this maximum flux, the effective $A_{\text {ratio }}$ could be increased. Such design modifications, in this case, could include increasing the length and taper of the entrance region, or changing the reflective properties inside the entrance region to reflect more rays into the body of the tube. After these design modifications are made, ray tracing can be used to evaluate the new effective $A_{\text {ratio }}$ and absorption efficiency of the receiver, and then determine the new $h_{h t f}$ requirements to meet the desired thermal efficiency.

\section{Summary and Conclusions}

This research has developed a simplified method for analyzing a cavity-based CSP receiver design. This technique can be coupled with high accuracy techniques, such as Monte Carlo ray tracing and CFD, to increase the accuracy of the technique. Simplifying the overall analysis technique allows for the receiver design to be analyzed quickly, thus allowing for design optimization. Likewise, this technique allows for a broad quantitative comparison between designs without the need for a detailed analysis on the designs.

The simplified model provides a technique to quickly analyze the thermal performance of CSP receivers to guide detailed receiver design and engineering. Through this analysis technique:

1. Overall thermal efficiency can be calculated given inlet and outlet temperatures, $A_{\text {ratio }}$, maximum receiver operating temperature, and ranges for $h_{h t f}$ and $h_{a m b}$. 
[1] Z. Ma, G. Glatzmaier, M. Mehos, Fluidized Bed Technology for Concentrating Solar Power with Thermal Energy Storage, Journal of Solar Energy Engineering, 136 (2014).

[2] Z. Ma, G.C. Glatzmaier, C.F. Kutscher, Thermal Energy Storage and its Potential Applications in Solar Thermal Power Plants and Electricity Storage, ASME 2011 5th International Conference on Energy Sustainability, American Society of Mechanical Engineers, 2011, pp. 447-456.

[3] S.M. Flueckiger, B.D. Iverson, S.V. Garimella, Economic Optimization of a Concentrating Solar Power Plant with Molten-Salt Thermocline Storage, Journal of Solar Energy Engineering, 136 (2014).

[4] CSP Component Research and Development, http://energy.gov/eere/sunshot/csp-component-research-anddevelopment, March 2015.

[5] R.W. Bradshaw, D.B. Dawson, W. De La Rosa, R. Gilbert, S.H. Goods, M.J. Hale, P. Jacobs, S.A. Jones, G.J. Kolb, J.E. Pacheco, Final Test and Evaluation Results from the Solar Two Project, SAND2002-0120, Sandia National Laboratory, Albuquerque, NM (US), 2002.

[6] M. Romero, R. Buck, J.E. Pacheco, An Update on Solar Central Receiver Systems, Projects, and Technologies, Journal of Solar Energy Engineering, 124 (2002) 98-108.

[7] O. Behar, A. Khellaf, K. Mohammedi, A Review of Studies on Central Receiver Solar Thermal Power Plants, Renewable and Sustainable Energy Reviews, 23 (2013) 12-39.

[8] C.K. Ho, B.D. Iverson, Review of High-Temperature Central Receiver Designs for Concentrating Solar Power, Renewable and Sustainable Energy Reviews, 29 (2014) 835-846.

[9] A.L. Avila-Marin, Volumetric Receivers in Solar Thermal Power Plants with Central Receiver System Technology: a Review, Solar Energy, 85 (2011) 891-910.

[10] A. Fleming, Z. Ma, T. Wendelin, H. Ban, C. Folsom, Thermal Modeling of a Multi-Cavity Array Receiver Performance for Concentrating Solar Power Generation, ASME 2015 9th International Conference on Energy 
Sustainability and 13th International Conference on Fuel Cell Science, Engineering and Technology, American Society of Mechanical Engineers, San Diego, CA, 2015.

[11] J. Martinek, Z. Ma, Granular Flow and Heat Transfer Study in a Near-Blackbody Enclosed Particle Receiver, ASME 2014 8th International Conference on Energy Sustainability collocated with the ASME 2014 12th

International Conference on Fuel Cell Science, Engineering and Technology, American Society of Mechanical Engineers, 2014.

[12] A. Carotenuto, F. Reale, G. Ruocco, U. Nocera, F. Bonomo, Thermal Behaviour of a Multi-Cavity Volumetric Solar Receiver: Design and Tests Results, Solar Energy, 50 (1993) 113-121.

[13] Near-Blackbody Enclosed Particle Receiver, http://energy.gov/sites/prod/files/2014/01/f7/csp_review_meeting_042413_ma.pdf, March 2015.

[14] J.M. Christian, C.K. Ho, CFD Simulation and Heat Loss Analysis of the Solar Two Power Tower Receiver, ASME 2012 6th International Conference on Energy Sustainability collocated with the ASME 2012 10th International Conference on Fuel Cell Science, Engineering and Technology, American Society of Mechanical Engineers, 2012, pp. 227-235.

[15] M. Prakash, Numerical Studies on Natural Convection Heat Losses from Open Cubical Cavities, Journal of Engineering, 2013 (2013).

[16] S.M. Besarati, D.Y. Goswami, E.K. Stefanakos, Optimal Heliostat Aiming Strategy for Uniform Distribution of Heat Flux on the Receiver of a Solar Power Tower Plant, Energy Conversion and Management, 84 (2014) $234-243$.

[17] F. García-Martín, M. Berenguel, A. Valverde, E. Camacho, Heuristic Knowledge-based Heliostat Field Control for the Optimization of the Temperature Distribution in a Volumetric Receiver, Solar Energy, 66 (1999) 355-369.

[18] A.J. Gallego, F. Fele, E.F. Camacho, On the Optimization of Irradiance Distribution in Solar Tower Plants with Flat Receivers, Control Conference (ECC), 2014 European, IEEE, 2014, pp. 2496-2501.

[19] A. Sánchez-González, D. Santana, Solar Flux Distribution on Central Receivers: A Projection Method from Analytic Function, Renewable Energy, 74 (2015) 576-587.

[20] T. Wendelin, SolTRACE: A New Optical Modeling Tool for Concentrating Solar Optics, ASME 2003

International Solar Energy Conference, American Society of Mechanical Engineers, 2003, pp. 253-260.

[21] C. Singer, R. Buck, R. Pitz-Paal, H. Müller-Steinhagen, Assessment of Solar Power Tower Driven

Ultrasupercritical Steam Cycles Applying Tubular Central Receivers with Varied Heat Transfer Media, Journal of Solar Energy Engineering, 132 (2010).

[22] S. Paitoonsurikarn, K. Lovegrove, A New Correlation for Predicting the Free Convection Loss from Solar Dish Concentrating Receivers, In Proceedings of the 44th Annual Conference of the Australian and New Zealand Solar Energy Society, 2006.

[23] X. Li, W. Kong, Z. Wang, C. Chang, F. Bai, Thermal Model and Thermodynamic Performance of Molten Salt Cavity Receiver, Renewable Energy, 35 (2010) 981-988.

[24] R. Jilte, S. Kedare, J. Nayak, Natural Convection and Radiation Heat Loss from Open Cavities of Different Shapes and Sizes used with Dish Concentrator, Mechanical Engineering Research, 3 (2013) 25.

[25] J.K. Yuan, C.K. Ho, J.M. Christian, Numerical Simulation of Natural Convection in Solar Cavity Receivers, Journal of Solar Energy Engineering, 137 (2015).

[26] T. Taumoefolau, S. Paitoonsurikarn, G. Hughes, K. Lovegrove, Experimental Investigation of Natural Convection Heat Loss from a Model Solar Concentrator Cavity Receiver, Journal of Solar Energy Engineering, 126 (2004) 801-807.

[27] A. Clausing, Convective Losses from Cavity Solar Receivers - Comparisons between Analytical Predictions and Experimental Results, Journal of Solar Energy Engineering, 105 (1983) 29-33.

[28] A. Clausing, An Analysis of Convective Losses from Cavity Solar Central Receivers, Solar Energy, 27 (1981) 295-300.

[29] P.K. Falcone, A Handbook for Solar Central Receiver Design, SAND-86-8009, Sandia National Labs., Livermore, CA (USA), 1986.

[30] H. Al-Ansary, A. El-Leathy, Z. Al-Suhaibani, S. Jeter, D. Sadowski, A. Alrished, M. Golob, Experimental Study of a Sand-air Heat Exchanger for use with a High-Temperature Solar Gas Turbine System, Journal of Solar Energy Engineering, 134 (2012).

[31] R. Pitz-Paal, B. Hoffschmidt, M. Böhmer, M. Becker, Experimental and Numerical Evaluation of the Performance and Flow Stability of Different Types of Open Volumetric Absorbers under Non-homogeneous Irradiation, Solar Energy, 60 (1997) 135-150.

[32] M. Golob, S. Jeter, D. Sadowski, Heat Transfer from Flat Surfaces to Moving Sand, 2010 ASME Early Career Technical Conference, Atlanta, GA, 2010.

[33] F.P. Incropera, Fundamentals of Heat and Mass Transfer, John Wiley \& Sons, 2011. 
2

3

4

5

6

7

8

9

10

11

12

13

14

15

16

17

18

19

20

21

22

23

24

25

26

27

28

29

30

31

32

33

34

35

36

37

38

39

40

41

42

43

44

45

46

47

48

49

50

51

52

53

54

55

56

57

58

59

60

61

62

63

64

65

$1 \quad$ Figure 1. Diagram of control volume with energy and mass balance used in model for thermal efficiency calculation. 
$1 \quad$ Figure 2. Local efficiency versus local HTF temperature for three $h A_{\text {ratio }}$ cases $\left(h_{\text {amb }}=25 \mathrm{~W} \cdot \mathrm{m}^{-2} \cdot \mathrm{K}^{-1}, \eta_{\text {absorb }}=0.98, \varepsilon_{\text {eff }}\right.$ $\left.2=1, q_{\text {aper }}^{\prime \prime}=1 \mathrm{MW} / \mathrm{m}^{2}\right)$. While the local efficiency curves will change with the aperture flux, the curves are always 3 monotonically decreasing with temperature. 
50

51 
1 Figure 4. Receiver overall efficiency for a range of $h A_{\text {ratio }}$ and flux values given inlet and outlet temperatures of $2400 K$ and $1000 K$. Provided for three different values for convection coefficients to the environment of $h_{a m b}=0,25$, 3 and $50 \mathrm{~W} \cdot \mathrm{m}^{-2} \cdot \mathrm{K}^{-1}$ and assuming a uniform aperture flux on the receiver $\left(\eta_{\text {absorb }}=0.98, \varepsilon_{\text {eff }}=1\right)$. 
1 Figure 5. Optimizing the flux at all locations within the receiver yields these receiver temperatures, fluxes, and local 2 efficiencies plots. The overall efficiency for this case is $89.72 \%$. $\left(h_{a m b}=25 \mathrm{~W} \cdot \mathrm{m}^{-2} \cdot \mathrm{K}^{-1}, \eta_{\text {absorb }}=0.98, \varepsilon_{\text {eff }}=1\right.$, $3 h A_{\text {ratio }}=3000 \mathrm{~W} \cdot \mathrm{m}^{-2} \cdot \mathrm{K}^{-1}$ ) 
50

51
$1 \quad$ Figure 6. Receiver with a material temperature limitation of $1200 \mathrm{~K}$. Optimizing the flux at all locations within the 2 receiver yields these receiver temperatures, fluxes, and local efficiencies plots. The overall efficiency for this case is $389.57 \%$. $\left(h_{a m b}=25 \mathrm{~W} \cdot \mathrm{m}^{-2} \cdot \mathrm{K}^{-1}, \eta_{\text {absorb }}=0.98, \varepsilon_{\text {eff }}=1, h A_{\text {ratio }}=3000 \mathrm{~W} \cdot \mathrm{m}^{-2} \cdot \mathrm{K}^{-1}\right)$ 
$1 \quad$ Figure 7. Overall receiver efficiency contour plot for maximum receiver temperature and a hA ratio with the aperture 2 flux optimized. The input and output HTF temperatures were $400 \mathrm{~K}$ and $1000 \mathrm{~K}$. Provided for three different values 3 for convection coefficients to the environment of $h_{a m b}=0,25$, and $50 \mathrm{~W} \cdot \mathrm{m}^{-2} \cdot \mathrm{K}^{-1}\left(\eta_{\text {absorb }}=0.98, \varepsilon_{e f f}=1\right)$. 


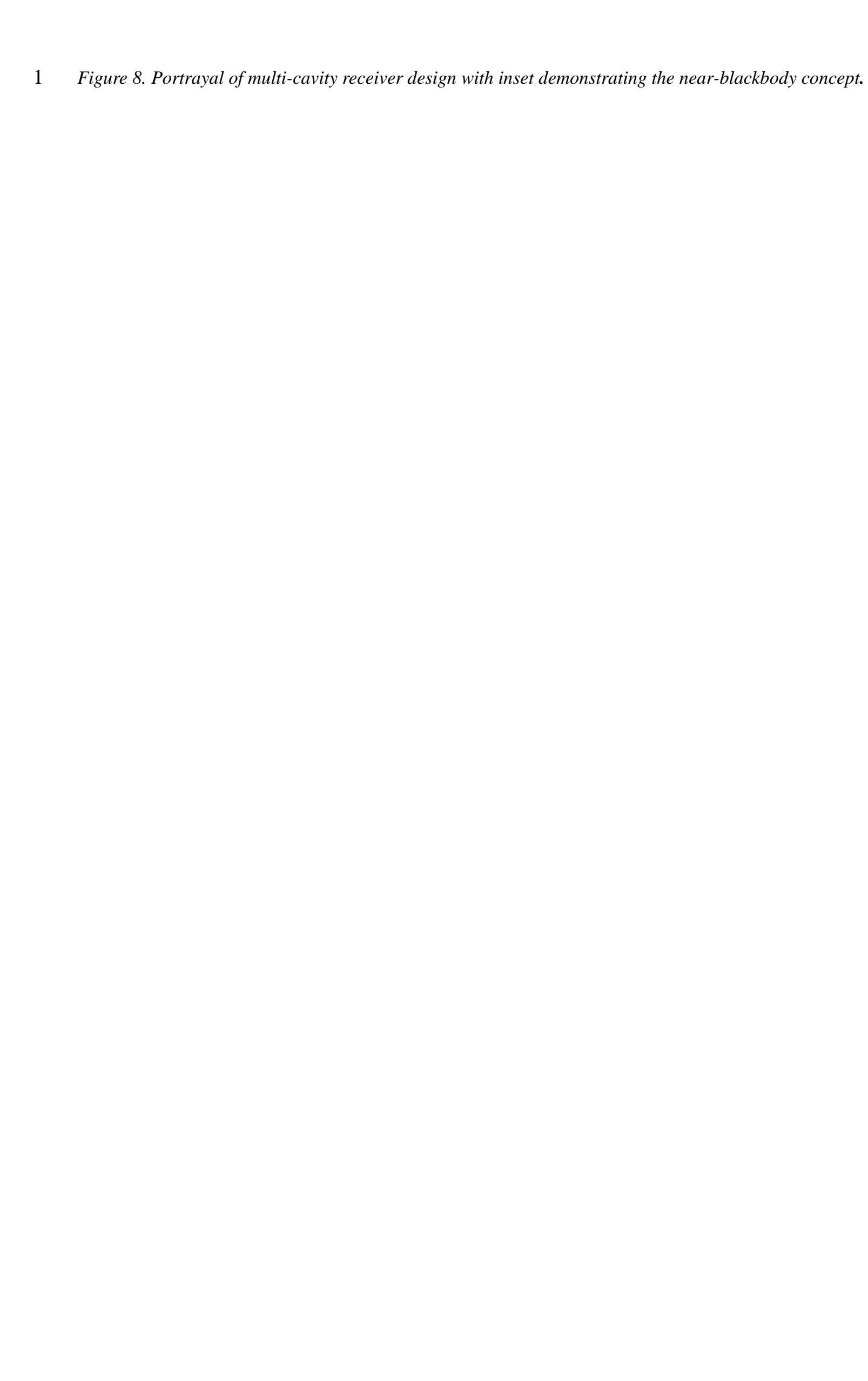


2

3

4

5

6

7

8

9

10

11

12

13

14

15

16

17

18

19

20

21

22

23

24

25

26

27

28

29

30

31

32

33

34

35

36

37

38

39

40

41

42

43

44

45

46

47

48

49

50

51

52

53

54

55

56

57

58

59

60

61

62

63

64

65

1 Figure 9. Flux distribution generated using SolTrace on three channels of the multi-cavity receiver. The flux at the 2 aperture of the receiver is $1.27 \mathrm{MW} / \mathrm{m}^{2}$. 
2

3

4

5

6

7

8

9

10

11

12

13

14

15

16

17

18

19

20

21

22

23

24

25

26

27

28

29

30

31

32

33

34

35

36

37

38

39

40

41

42

43

44

45

46

47

48

49

50

51

52

53

54

55

56

57

58

59

60

61

62

63

64

65

1 Figure 10. Ray tracing results display $>97 \%$ absorption efficiency can be obtained over a range of material 2 reflectivity values and specularity errors. 
2

3

4

5

6

7

8

9

10

11

12

13

14

15

16

17

18

19

20

21

22

23

24

25

26

27

28

29

30

31

32

33

34

35

36

37

38

39

40

41

42

43

44

45

46

47

48

49

50

51

52

53

54

55

56

57

58

59

60

61

62

63

64

65

1 Figure 11. Quality of flux spreading throughout the cavity represented by the ratio of the aperture flux to the 2 maximum flux. 
50

51

52 
Figure 1

Heat Transfer Fluid $\left(\dot{m}, \rho, C_{P}, T_{h t f, i n}\right)$
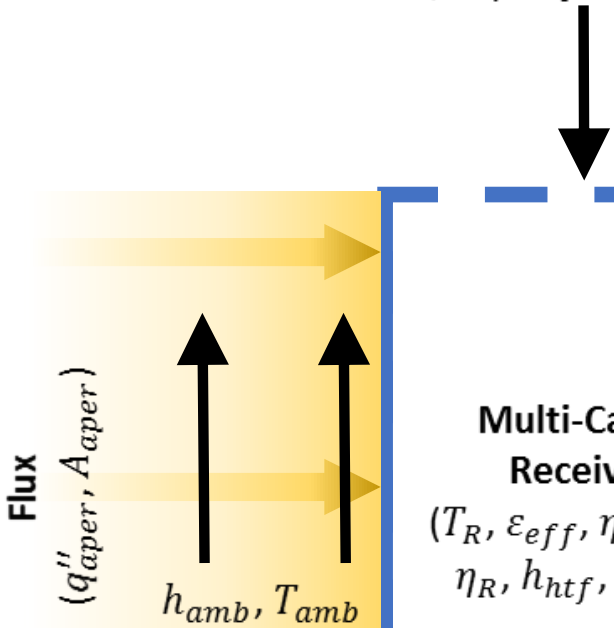

Multi-Cavity Receiver

$\left(T_{R}, \varepsilon_{\text {eff }}, \eta_{\text {absorb }}\right.$, $\left.\eta_{R}, h_{h t f}, A_{h t f}\right)$ 
Figure 2

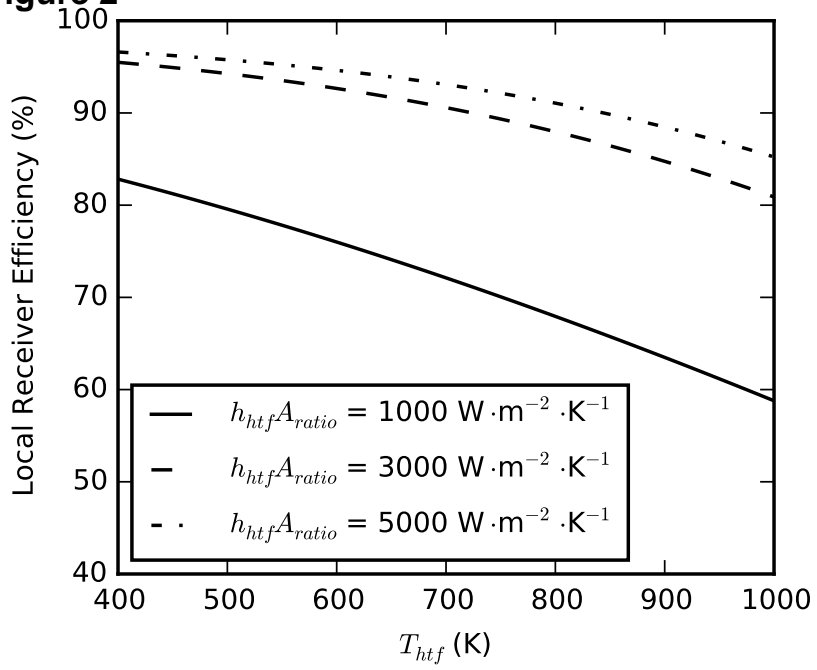


Figure 3
5000

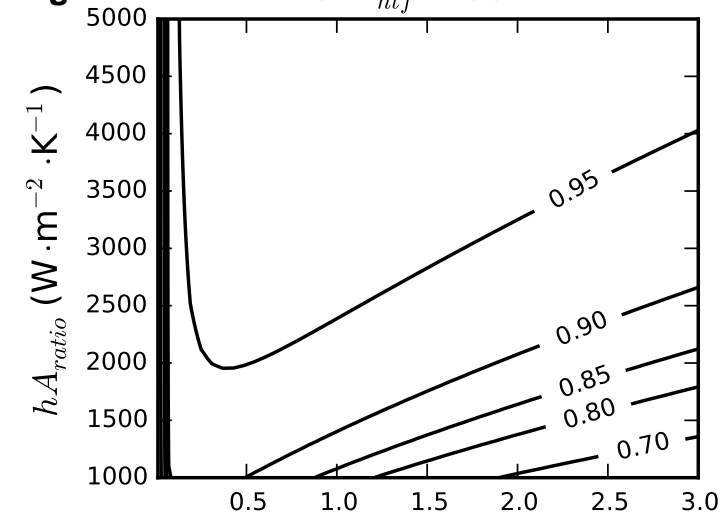

Flux $\left(\mathrm{MW} / \mathrm{m}^{2}\right)$ b. $T_{h t f}=700 \mathrm{~K}$

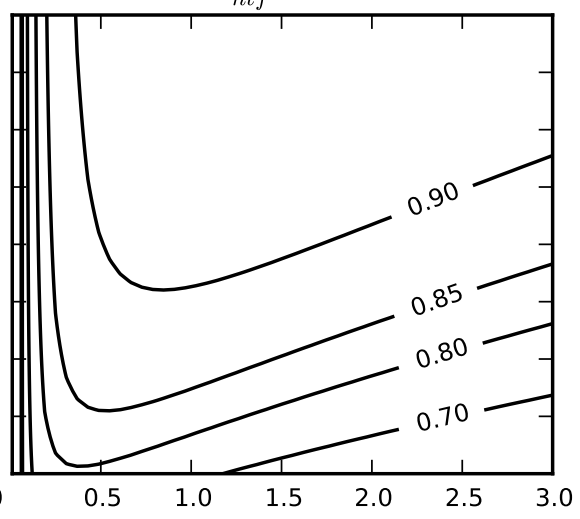

Flux $\left(\mathrm{MW} / \mathrm{m}^{2}\right)$ c. $T_{h t f}=1000 \mathrm{~K}$

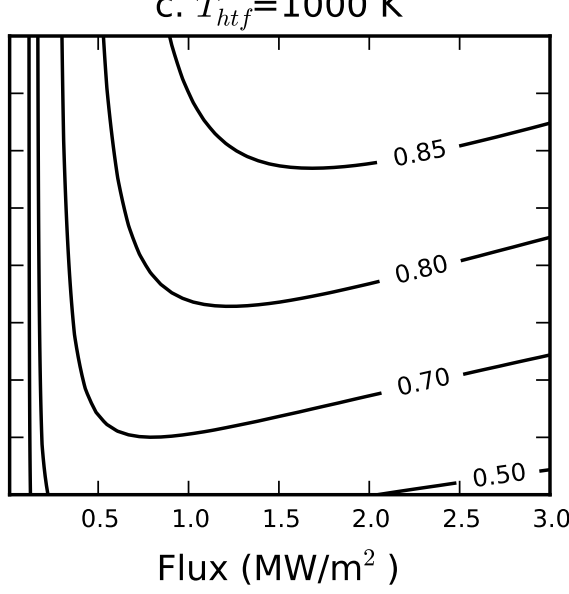


Figure 4

a. $h_{a m b}=0 \mathrm{~W} \cdot \mathrm{m}^{-2} \cdot \mathrm{K}^{-1}$

b. $h_{a m b}=25 \mathrm{~W} \cdot \mathrm{m}^{-2} \cdot \mathrm{K}^{-1}$

C. $h_{a m b}=50 \mathrm{~W} \cdot \mathrm{m}^{-2} \cdot \mathrm{K}^{-1}$

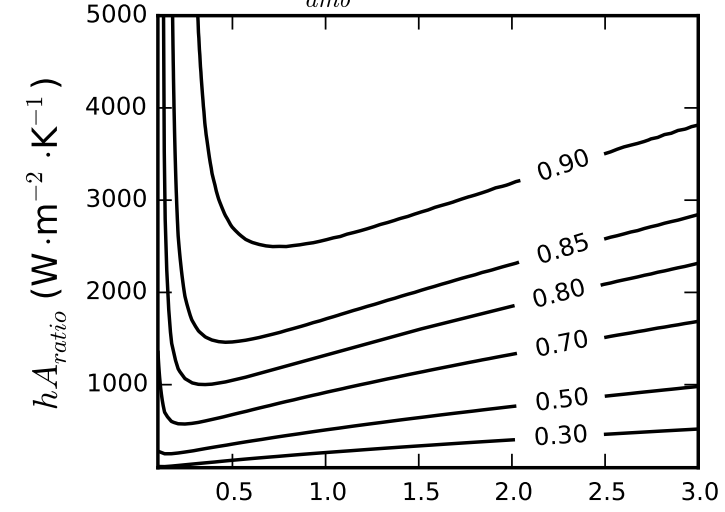

Flux $\left(\mathrm{MW} / \mathrm{m}^{2}\right)$

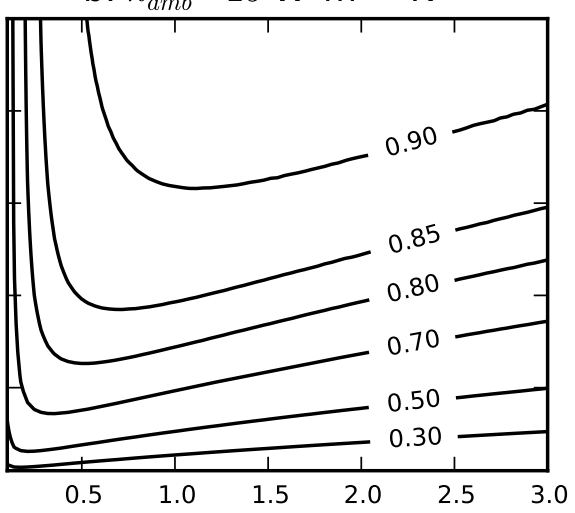

Flux $\left(\mathrm{MW} / \mathrm{m}^{2}\right)$

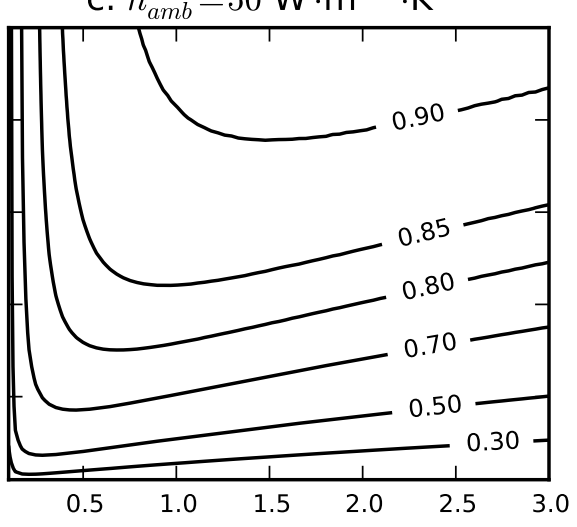

Flux $\left(\mathrm{MW} / \mathrm{m}^{2}\right)$ 
Figure 5

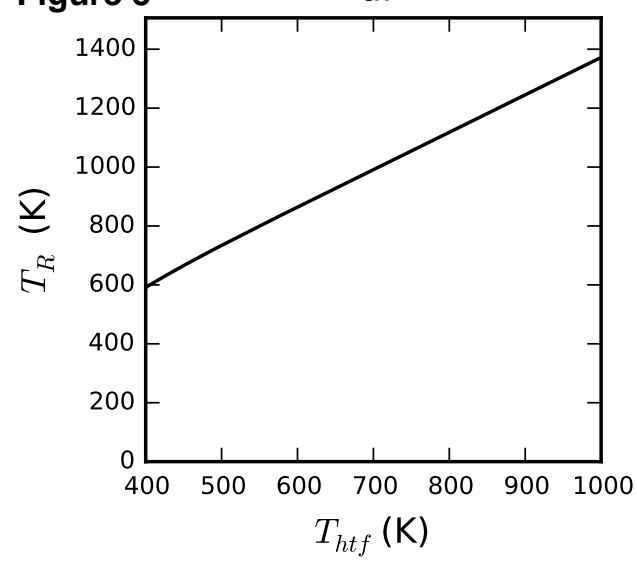

b.

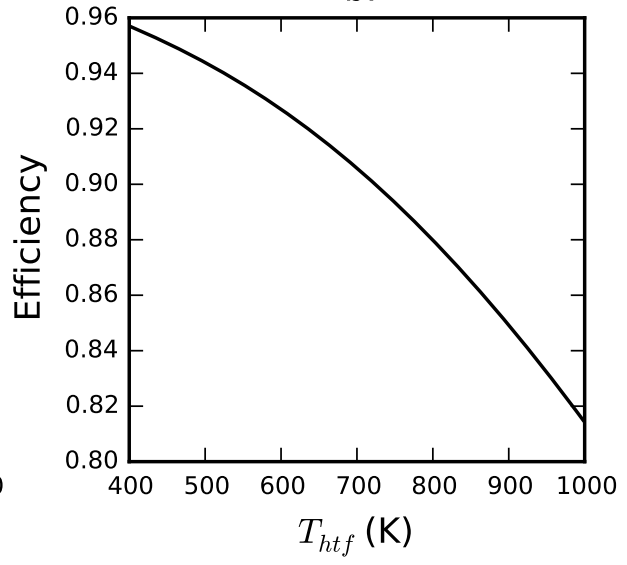

C.

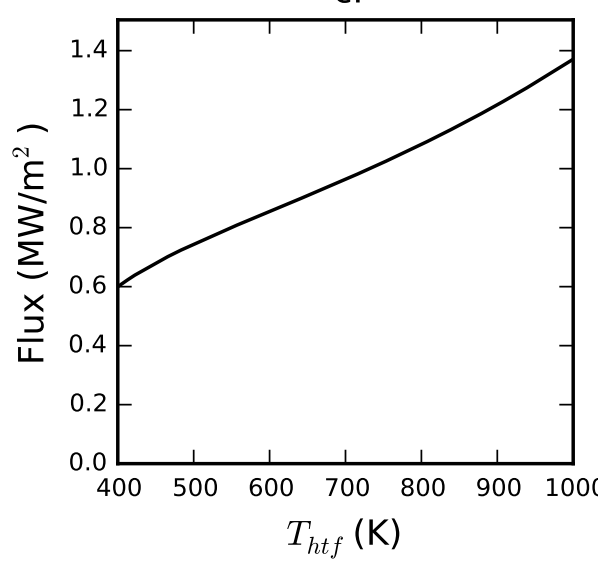


Figure 6

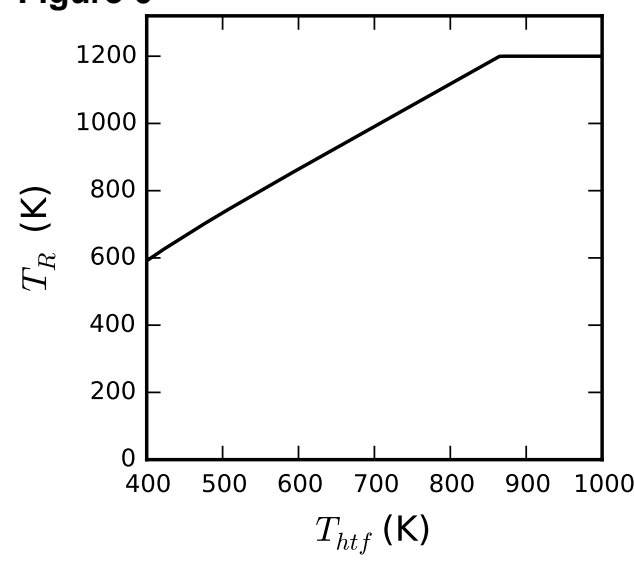

b.

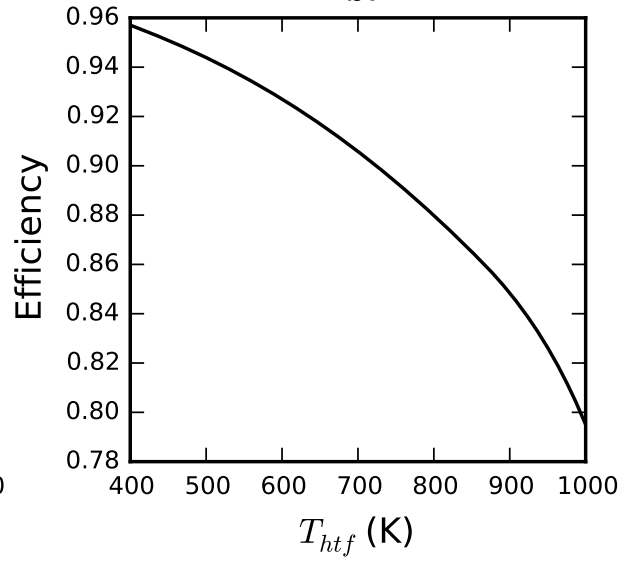

C.

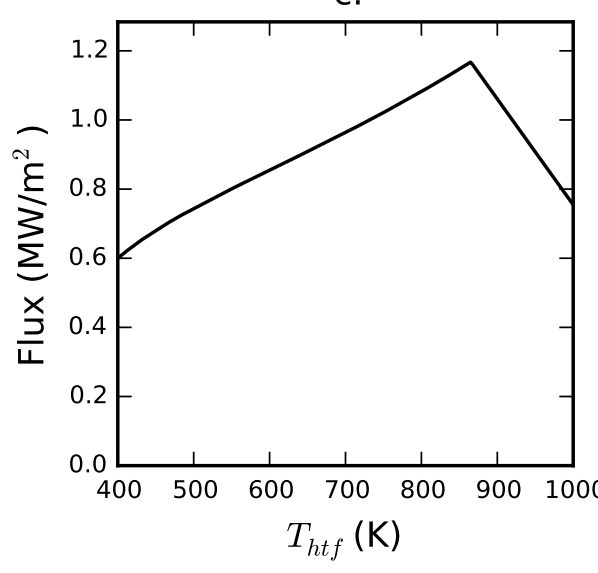


Figure 7

a. $h_{a m b}=0 \mathrm{~W} \cdot \mathrm{m}^{-2} \cdot \mathrm{K}^{-1}$

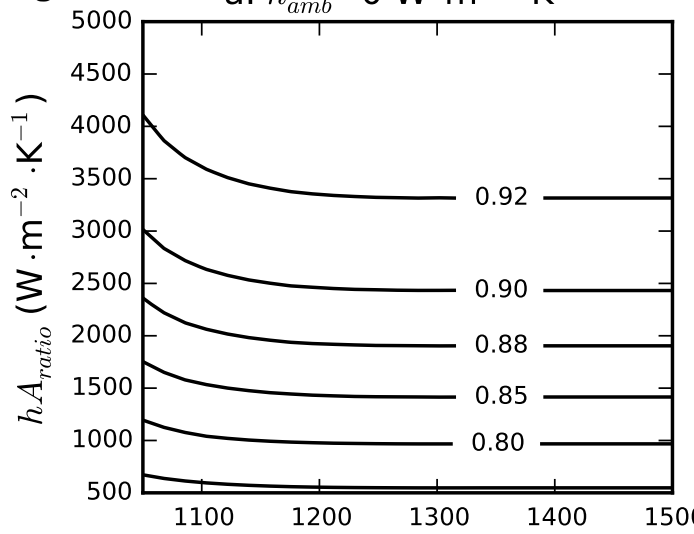

Max Receiver Temp (K) b. $h_{a m b}=25 \mathrm{~W} \cdot \mathrm{m}^{-2} \cdot \mathrm{K}^{-1}$

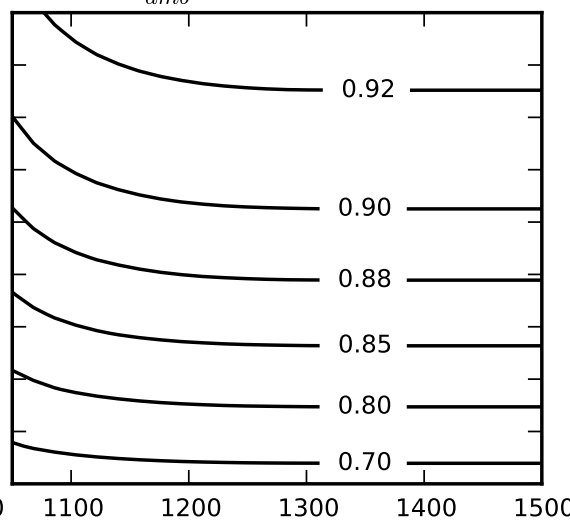

Max Receiver Temp (K) c. $h_{a m b}=50 \mathrm{~W} \cdot \mathrm{m}^{-2} \cdot \mathrm{K}^{-1}$

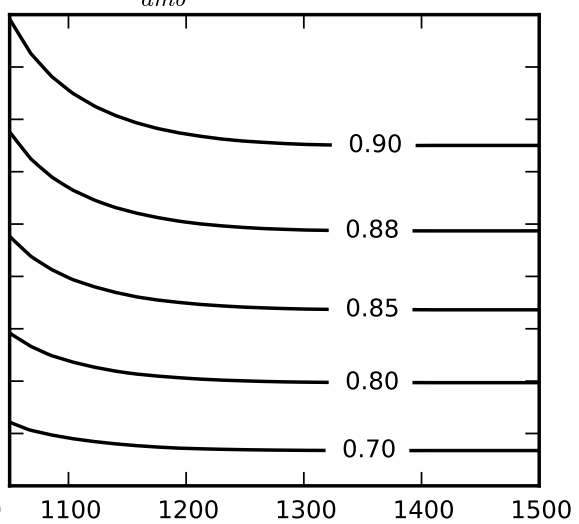

Max Receiver Temp (K) 


\section{Figure 8}

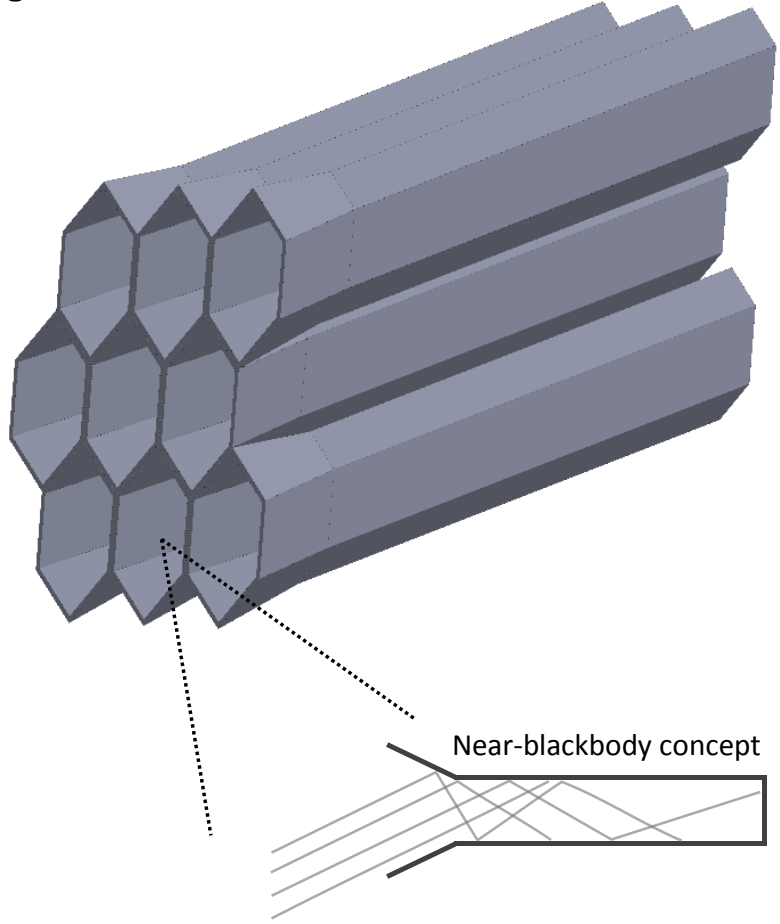




\section{Figure 9}

$2.5 e+005$

FLUX $(W / m \wedge 2)$
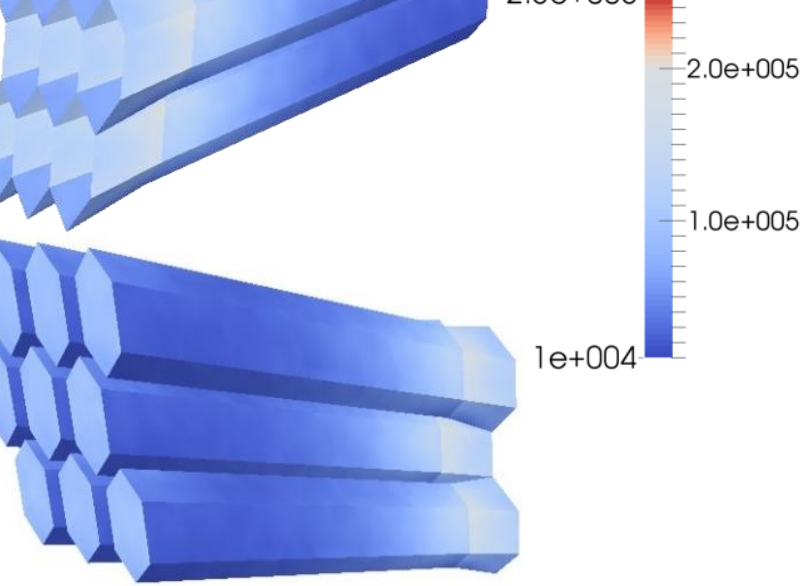


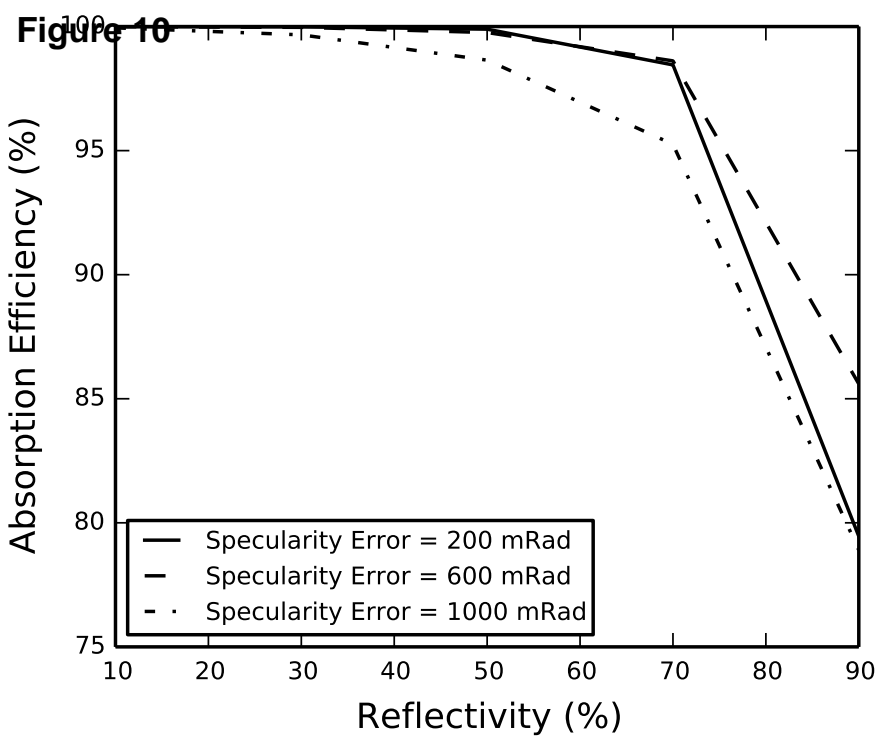




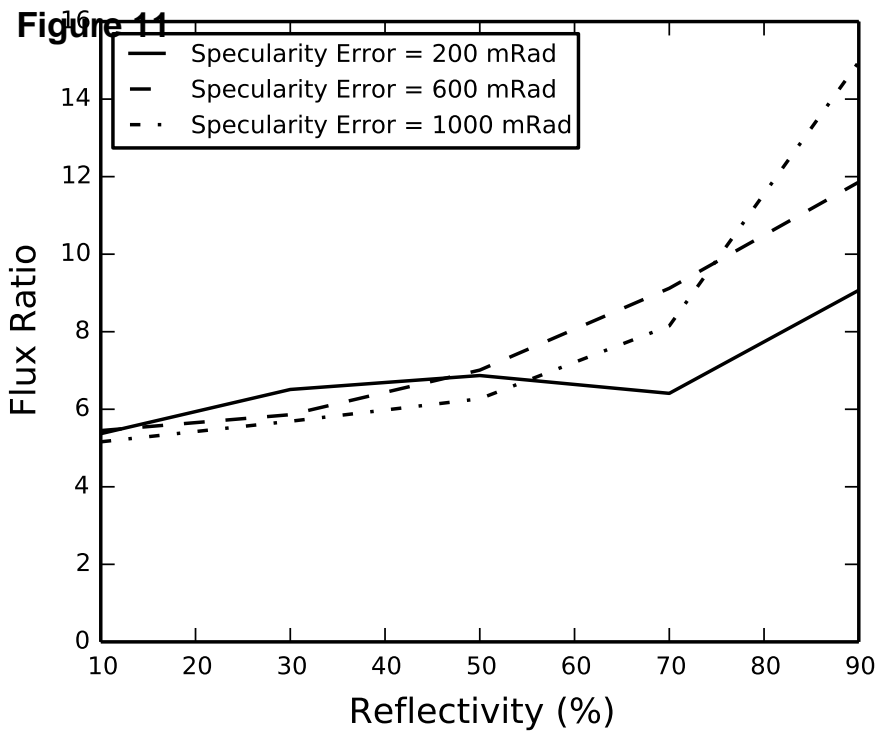


